\section{Case Reports in Oncology}

\title{
Successful Response to
}

Cyclophosphamide, Vincristine, and Dacarbazine Chemotherapy in a Patient with Metastatic Carotid Body Paraganglioma

\author{
Hiroki Yamada ${ }^{a}$ Toshirou Fukushima ${ }^{a}$ Takashi Kobayashi ${ }^{a}$ \\ Shintaro Kanda ${ }^{a}$ Tomonobu Koizumi $^{a}$ Mai Iwaya ${ }^{b}$ \\ aDepartment of Hematology and Medical Oncology, Shinshu University School of Medicine, \\ Asahi Matsumoto, Japan; ${ }^{b}$ Department of Laboratory Medicine, Shinshu University School \\ of Medicine, Asahi Matsumoto, Japan
}

Keywords

Pulmonary metastasis - Neuroendocrine tumor - Low-grade malignancy ·

Cyclophosphamide, vincristine, and dacarbazine chemotherapy · Paraganglioma

\begin{abstract}
Carotid body paraganglioma is a rare neuroendocrine tumor presenting with low-grade histological and clinical features. However, the tumor has the potential to produce distant metastasis, and due to its rarity, little information is available regarding chemotherapy for such metastatic lesions. Here, we report a case of carotid body paraganglioma with development of pulmonary and bone metastases 10 years after radical surgery for the primary lesion in the neck. The lesions showed a good response to cyclophosphamide, vincristine, and dacarbazine chemotherapy. A beneficial therapeutic outcome by chemotherapy is extremely rare in patients with metastatic carotid body paraganglioma.
\end{abstract}




\section{Introduction}

Head and neck paraganglioma, also known as chemodectoma [1], is an extremely rare neuroendocrine tumor arising from the paraganglia, which is estimated to account for $3 \%$ of all paragangliomas and $0.03 \%$ of all tumors [2, 3]. Carotid body paraganglioma (CBP) represents about $65 \%$ of all head and neck paragangliomas [2,3]. The clinical course of CBP is generally benign with slow growth, and localized CBP can be cured by surgical resection [4-10]. However, CBP has the potential to produce regional lymph node and/or distant metastases [5-8]. Systemic chemotherapy is indicated for the treatment of disseminated metastatic CBP. Due to its rarity, however, optimal chemotherapeutic strategies for metastatic CBP remain to be determined. Here, we report the clinical course of a patient with CBP who developed pulmonary and bone metastases 10 years after radical surgery for the primary tumor and showed a successful response to cyclophosphamide, vincristine, and dacarbazine (CVD) chemotherapy along with a review of the relevant literature.

\section{Case Presentation}

A 59-year-old man noticed a painless left cervical mass in 2003. He had a history of smoking (20 pack-years) and drinking alcohol (35 g ethanol/day) over 30 years, but no remarkable disease history. Aspiration cytology of the cervical mass showed no malignant findings, and he had been under observation. However, the cervical mass increased in size (Fig. 1a) and showed strong uptake on ${ }^{123}$ I-metaiodobenzylguanidine scintigraphy in 2010 (Fig. 1b). Total excision was performed, and a pathological diagnosis of CBP was made (Fig. 2a). He had been followed up in the outpatient clinic until 2013, after which the observation was discontinued. In 2020, he noticed gradually worsening back pain. There were no specific positive findings during physical examination including blood pressure $(126 / 76 \mathrm{~mm} \mathrm{Hg}$ ) and heart rate (68 beats per min). Routine laboratory test results on admission, including routine blood test results, coagulation test results, and levels of blood glucose, liver function, renal function, and serum tumor markers (carcinoembryonic antigen, alpha-fetoprotein, and carbohydrate antigen 19-9), were within normal ranges.

Spinal magnetic resonance imaging revealed bone metastases (Fig. 3a), and chest computed tomography revealed multiple intrapulmonary masses (Fig. 3a). ${ }^{18} \mathrm{~F}$-Fluorodeoxyglucose positron emission tomography ( ${ }^{18} \mathrm{~F}-\mathrm{FDG}$-PET) showed abnormal uptake of FDG in these metastatic lesions (Fig. 4). Bronchoscopic biopsy showed neuroendocrine tumor cells and the tumor cells positive for chromogranin A and synaptophysin on immunohistochemical staining confirming a diagnosis of pulmonary and bone metastases of CBP (Fig. 2b-d). Chemotherapy with cyclophosphamide $\left(750 \mathrm{mg} / \mathrm{m}^{2}\right.$, day 1), vincristine $\left(1.4 \mathrm{mg} / \mathrm{m}^{2}\right.$, day 1$)$, and dacarbazine $\left(600 \mathrm{mg} / \mathrm{m}^{2}\right.$, day 2$)$ (CVD) was performed with a 21-day cycle. Ten cycles of CVD therapy have been administered to date, and partial response has been achieved without any specific adverse events (Fig. 2b). The patient has not complained of lumbago without any specific treatment, and we are planning to continue the therapy.

Laboratory examination revealed that urinary secretion of vanillylmandelic acid decreased from $11.5 \mathrm{mg}$ /day (normal: $1.4-4.9 \mathrm{mg} /$ day) before chemotherapy to $4.8 \mathrm{mg} /$ day after chemotherapy. However, urinary 24-h metanephrine and normetanephrine excretion was similar before and after chemotherapy (metanephrine, from 0.32 to $0.24 \mu \mathrm{g} /$ day, normal $0.05-0.20 \mu \mathrm{g} /$ day; normetanephrine, from 0.36 to $0.48 \mu \mathrm{g} /$ day, normal $0.10-0.28 \mu \mathrm{g} / \mathrm{day}$, respectively). During the clinical course, he had no specific symptoms related to increased catecholamines, including headache, palpitation, and skin rash. 


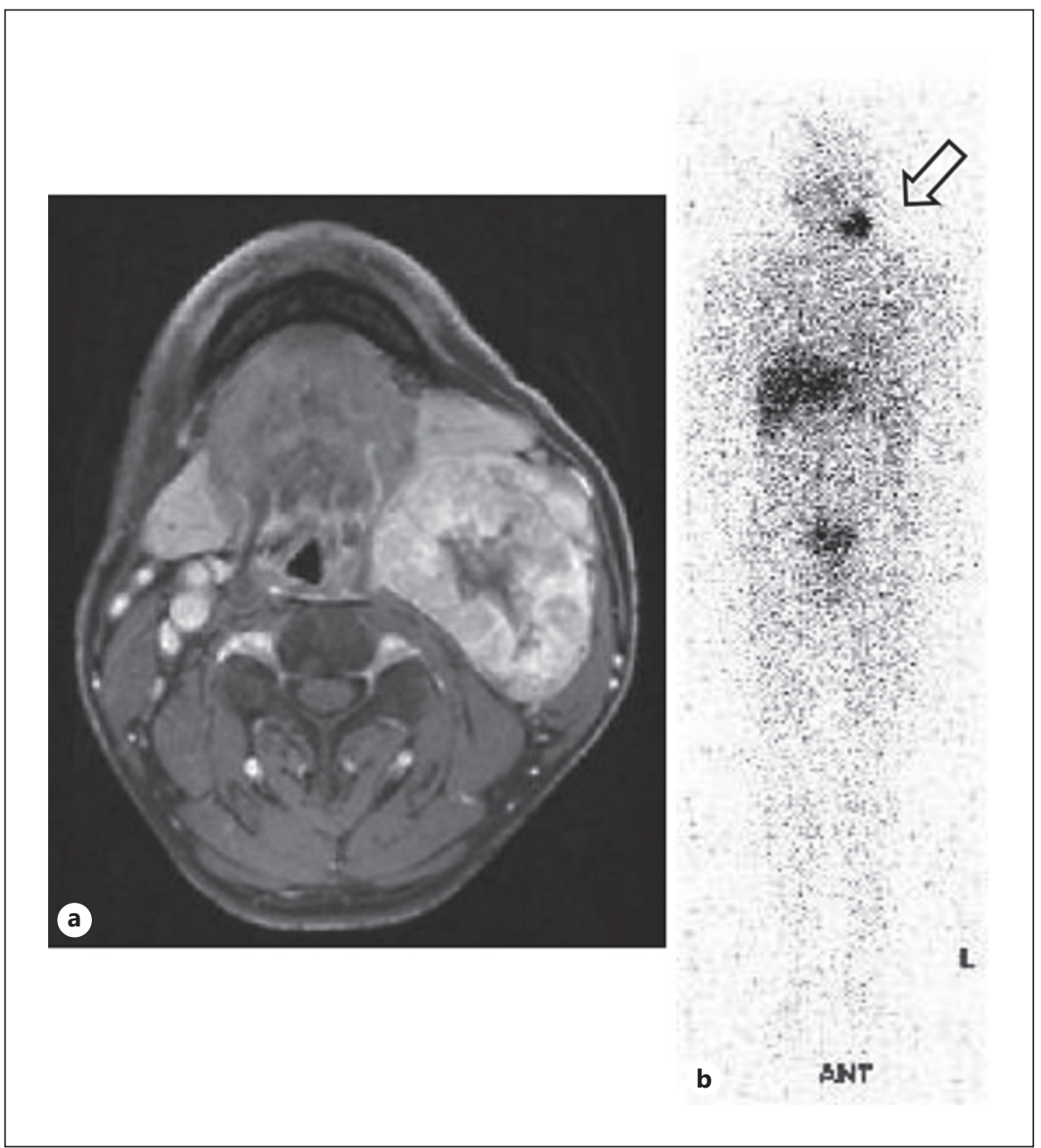

Fig. 1. a Contrast-enhanced T1-weighted magnetic resonance imaging showed a well-defined mass with a diameter of $10 \mathrm{~cm}$ in the left cervical region, displacing the left internal and external carotid arteries. b $^{123}$ I-Metaiodobenzylguanidine scintigraphy showed hyperintensity in the left cervical region.

\section{Discussion}

For the chemotherapeutic management of disseminated metastatic CBPs, most evidence is based on studies on pheochromocytoma/paraganglioma [10-12]. Huang et al. [10] summarized 18 cases of malignant pheochromocytoma/paraganglioma treated with CVD and reported a response rate of $56 \%$. Asai et al. [12] also reported a disease control rate (over stable disease) of 48\% in malignant pheochromocytoma/paraganglioma patients and survival benefit in these patients compared with nonresponders. Therefore, CVD chemotherapy is a useful chemotherapy regimen in patients with pheochromocytoma/paraganglioma. However, only 1 patient with CBP was included in these studies, and the response to CVD was not described. Therefore, little information is available about the response to systemic chemotherapy in CBP. Xing et al. [7] reviewed 10 case reports published from 1981 to 2018 regarding systemic therapy for distant metastatic CBP. Although various types of chemotherapy were performed, 2 patients receiving CVD achieved stable and partial disease, respectively. No other patients showed even a partial response to other chemotherapeutic regimens. We also searched PubMed MEDLINE for articles regarding CBPs and/or head and neck paragangliomas from 


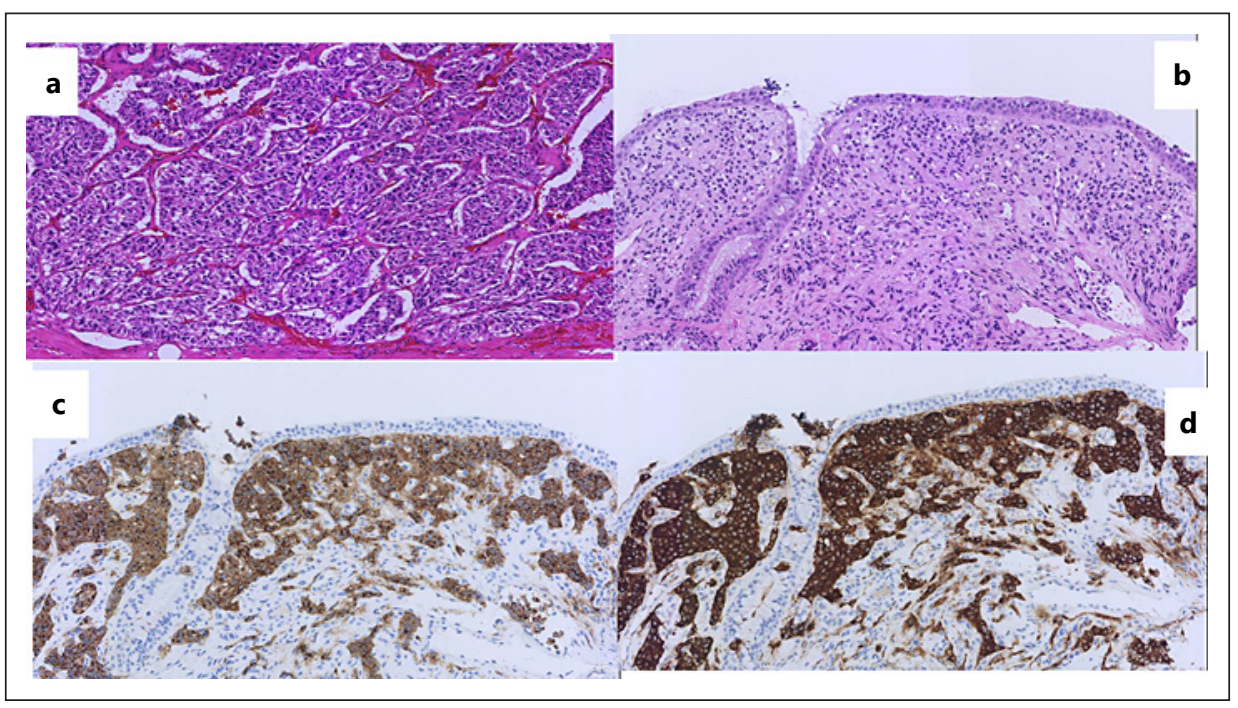

Fig. 2. a Pathological findings of resected neck tumor revealed abundant tumor cells in a solid nest (Zellballen pattern). $\mathbf{b}$ Pathological findings of the transbronchial biopsy specimens revealed dense proliferation of tumor cells with eosinophilic finely granular cytoplasm and round nuclei. The tumor cells were positive for chromogranin A (c) $(\times 156)$ and synaptophysin $(\mathbf{d})(\times 156)$ on immunohistological staining.

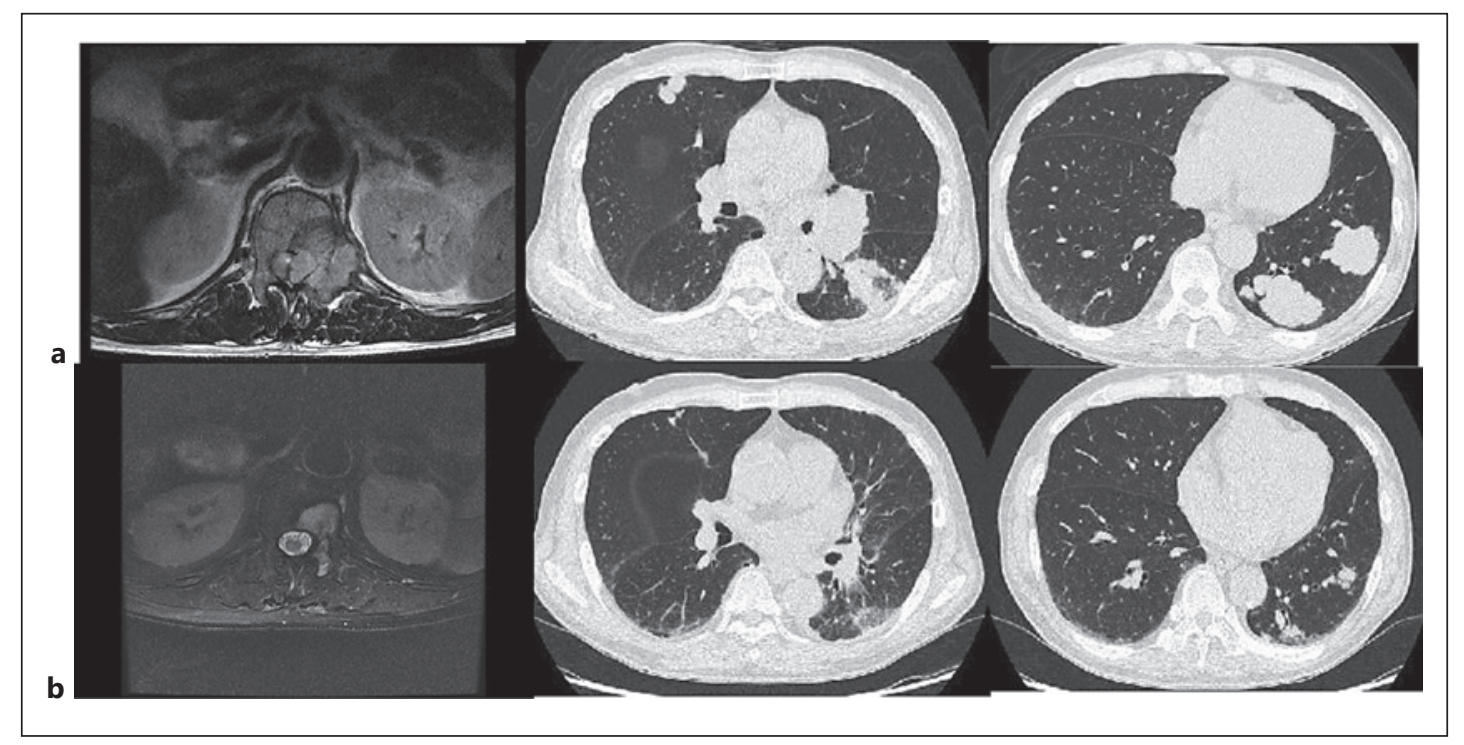

Fig. 3. a Before chemotherapy. Magnetic resonance imaging in the lumbar vertebrae showed phyllodes tumor occupying the vertebrae, and chest computed tomography revealed multiple metastatic nodules in both lungs. b After chemotherapy. The metastatic lesions were reduced after cyclophosphamide, vincristine, and dacarbazine chemotherapy.

2019 to date. However, no further information was found regarding efficacious chemotherapy for metastatic CBP. Our experience suggested that CVD chemotherapy could be useful in treatment of metastatic CBP.

With regard to molecular-based systemic therapy, sunitinib has been shown to be effective for malignant paragangliomas, especially in carriers of the $S D H B$ mutation [13]. Mutations in $S D H B, S D H C$, and $S D H D$ were shown to be associated with overexpression in angiogenesis and 
Fig. 4. 18F-Fluorodeoxyglucose positron emission tomography/computed tomography showed increased 18F-FDG uptake in the pulmonary and lumbar vertebrae.

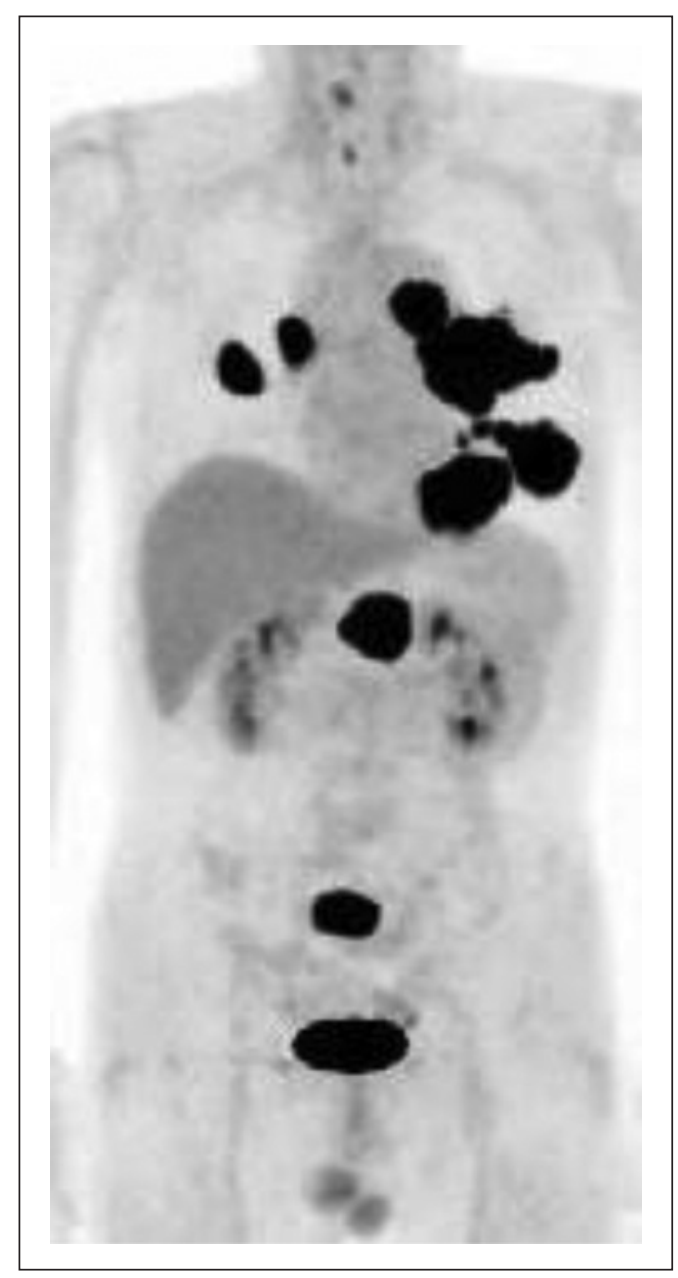

of angiogenic molecules, including vascular endothelial growth factor (VEGF) and VEGF receptor, in paraganglioma and pheochromocytoma [14]. To our knowledge, there have been no reports of the usefulness of antiangiogenic agents in CBP. Further information about comprehensive genomic profiling examination in CBP may contribute to the development of appropriate therapeutic regimens.

Based on the reported cases, metastatic sites of CBP include the lung, bone, liver, pancreas, and thyroid $[8,11-13]$. The average time to distant metastasis was 10.3 years after initial diagnosis [7]. The involved lesions and the time to distant metastasis in our case were consistent with those in previous reports. We would like to emphasize that although the incidence of metastasis is rare, long-term follow-up is necessary because of the possibility of disease recurrence many years after primary tumor resection in patients with CBP.

\section{Conclusion}

Little information is available for useful chemotherapy for metastatic CBP. The present case suggests that CVD chemotherapy is efficacious in metastatic CBP. Further clinical experience and studies are required to confirm our findings. 


\section{Case Reports in Oncology}

\begin{tabular}{l|l}
\hline Case Rep Oncol 2021;14:1827-1833 \\
\hline DOI: 10.1159/000520851 & $\begin{array}{l}\text { @ 2021 The Author(s). Published by S. Karger AG, Basel } \\
\text { www.karger.com/cro }\end{array}$ \\
\hline
\end{tabular}

Yamada et al.: Successful Response of Metastatic CBP to CVD Chemotherapy

\section{Statement of Ethics}

This study was conducted in accordance with the World Medical Association Declaration of Helsinki and was exempt from ethics committee approval because it is only 1 case report. Written informed consent was obtained from the patient for publication of this case report and any accompanying images.

\section{Conflict of Interest Statement}

None of the authors have any relevant financial relationships with a commercial interest.

\section{Funding Sources}

The authors did not receive any funding.

\section{Author Contributions}

Hiroki Yamada, Toshirou Fukushima, Takashi Kobayashi, Shintaro Kanda, and Tomonobu Koizumi treated and followed the patient. Mai Iwaya was responsible for the pathological analysis and interpretation. Hiroki Yamada collected the relevant clinical data of the present case and wrote the manuscript. Tomonobu Koizumi checked the manuscript and provided suggestions for revision. All authors contributed equally to this work and have read and approved the final manuscript.

\section{Data Availability Statement}

All data generated or analyzed during this study are included in this published article. Further enquiries can be directed to the corresponding author.

\section{References}

1 Boedeker CC, Ridder GJ, Schipper J. Paragangliomas of the head and neck: diagnosis and treatment. Fam Cancer. 2005;4:55-9.

2 Taïeb D, Kaliski A, Boedeker CC, Martucci V, Fojo T, Adler JR, et al. Current approaches and recent developments in the management of head and neck paragangliomas. Endocrine Rev. 2014;35:795-819.

3 Lee JH, Barich F, Karnell LH, Robinson RA, Zhen WK, Gantz BJ, et al. National Cancer Data Base report on malignant paragangliomas of the head and neck. Cancer. 2002;94:730-7.

4 Prasad SC, Paties CT, Pantalone MR, Mariani-Costantini R, Sanna M. Carotid body and vagal paragangliomas: epidemiology, genetics, clinicopathological features, imaging, and surgical management. In: Mariani-Costantini R, editor. Paraganglioma: a multidisciplinary approach [Internet]. Brisbane,AU: Codon Publications; 2019. Chapter 5.

5 Luna-Ortiz K, Rascon-Ortiz M, Villavicencio-Valencia V, Granados-Garcia M, Herrera-Gomez A. Carotid body tumors: review of a 20-year experience. Oral Oncol. 2005;41:56-61.

6 Fathalla AE, Elalfy MA. Clinical outcome of carotid body paraganglioma management: a review of 10-year experience. J Oncol. 2020;2020:6081273.

7 Xing J, Cheng Y, Ying H, Guan M, Jia N, Bai C. Systemic treatment of a metastatic carotid body tumor: a case report and literature review. Medicine. 2020;99:e22811.

8 Nishijima H, Asakage T, Sugasawa M. Malignant carotid body tumor with systemic metastases. Ann Otol Rhinol Laryngol. 2011;120:381-5.

9 Pacheco-Ojeda L. Malignant carotid body tumors: report of three cases. Ann Otol Rhinol Laryngol. 2001;110:36-40.

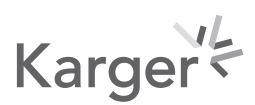


10 Huang H, Abraham J, Hung E, Averbuch S, Merino M, Steinberg SM, et al. Treatment of malignant pheochromocytoma/paraganglioma with cyclophosphamide, vincristine, and dacarbazine: recommendation from a 22-year follow-up of 18 patients. Cancer. 2008;113:2020-8.

11 Tanabe A, Naruse M, Nomura K, Tsuiki M, Tsumagari A, Ichihara A. Combination chemotherapy with cyclophosphamide, vincristine, and dacarbazine in patients with malignant pheochromocytoma and paraganglioma. Horm Cancer. 2013;4:103-10.

12 Asai S, Katabami T, Tsuiki M, Tanaka Y, Naruse M. Controlling tumor progression with cyclophosphamide, vincristine, and dacarbazine treatment improves survival in patients with metastatic and unresectable malignant pheochromocytomas/paragangliomas. Horm Cancer. 2017;8:108-18.

13 Ayala-Ramirez M, Chougnet CN, Habra MA, Palmer JL, Leboulleux S, Cabanillas ME, et al. Treatment with sunitinib for patients with progressive metastatic pheochromocytomas and sympathetic paragangliomas. J Clin Endocrinol Metab. 2012;97:4040-50.

14 Greenberg SE, Holman R, Kohlmann W, Buchmann L, Naumer A. Paraganglioma and other tumour detection rates in individuals with SDHx pathogenic variants by age of diagnosis and after the age of 50. Clin Endocrinol. 2021;95(3):447-52. 